\title{
Outcome of universal newborn eye screening with wide-field digital retinal image acquisition system: a pilot study
}

P Goyal' ${ }^{1}$, TR Padhi ${ }^{1}$, T Das ${ }^{2}$, L Pradhan ${ }^{3}$, S Sutar' ${ }^{1}$, S Butola ${ }^{1}$, UC Behera ${ }^{1}$, L Jain ${ }^{1}$ and S Jalali

\begin{abstract}
Purpose To evaluate the outcome of universal newborn eye screening with widefield digital retinal imaging (WFDRI) system. Methods In this pilot study, we examined 1152 apparently healthy newborn infants in the obstetrics and gynecology ward of a civil hospital in Eastern India over 1.5 years. The examination included external eye examination, red reflex test and fundus imaging by WFDRI (RetCam II, Clarity medical system, Pleasanton, CA, USA) by a trained optometrist. The pathologies detected, net monetary gain and skilled manpower saved were documented. The results were compared with three similar studies thus far published in the literature.

Results Ocular abnormality of any kind was seen in 172 (14.93\%) babies. Retinal hemorrhage in 153 babies $(88.9 \%$ of all abnormal findings) was the most common abnormality; it was bilateral in 118 (77.12\%) babies and 4 babies had foveal hemorrhage. Other abnormalities included vitreous hemorrhage $(n=1)$, congenital glaucoma $(n=2)$, uveal coloboma $(n=2)$, retinopathy

detecting treatment warranting ocular pathologies.

Eye (2018) 32, 67-73; doi:10.1038/eye.2017.129; published online 24 July 2017

\section{Introduction}

Universal newborn eye screening is an emerging concept for early intervention of many eye diseases that present at birth.

Photodocumentation by a technician and analysis by a remote expert has already proven to be a cost effective strategy in the early detection of diseases like retinopathy of prematurity $^{1}$ and diabetic retinopathy. ${ }^{2,3}$ In a pilot project we followed a technique of photographic documentation of the external and internal structures of the eye of a newborn by a trained technician and validation by an ophthalmologist later. The purpose was to analyze the outcome of this universal newborn eye screening with wide-field digital retinal imaging (WFDRI), assess the cost-benefit margin and compare it with few similar studies reported so far in the literature.
\end{abstract} mimicking retinopathy of prematurity $(n=2)$, and cystic fovea $(n=3)$. The retinal hemorrhages resolved spontaneously in all eyes. One baby with congenital glaucoma received surgery and the other was treated medically. The benefits included savings in skilled manpower, a net monetary gain of INR 4.195 million (US\$ 62,612) and skilled manpower saving by $319.4 \mathrm{~h}$.

Conclusions The universal neonatal eye screening using WFDRI detected pathologies that needed immediate care or regular follow up; saved skilled manpower with a net monetary gain. But compared to a red reflex test the benefits were marginal in terms of

\section{Materials and methods}

We designed a universal newborn baby screening protocol at the obstetrics and gynecology service of a civil hospital in Bhubaneswar, Eastern India from March 2014 to Oct 2015 (1.5 years). The hospital serves over 1 million population at Bhubaneswar (Capital city of Odisha in Eastern India) and people of all socioeconomic status from adjacent districts. Majority of the population served belong to a uniform racial distribution (Asian Indians). In general, the newborns are hospitalized for $24 \mathrm{~h}$ after vaginal delivery and for 3-4 days after $72 \mathrm{~h}$
${ }^{1}$ Retina and Vitreous services, Shri Mithu Tulsi Chanrai Campus, LV Prasad Eye Institute, Bhubaneswar, India

${ }^{2}$ Smt. Kanuri Santhamma Center for Vitreoretinal Services, Kallam Anji Reddy Campus, LV Prasad Eye Institute, Hyderabad, India

${ }^{3}$ Infosys Pediatric Center, Capital Hospital,

Bhubaneswar, India

Correspondence:

TR Padhi, Faculty, Vitreoretinal services, LV Prasad Eye Institute, Patia, Bhubaneswar 751024, India Tel: +91 9438361078; Fax: +91674398 7130 . E-mail: tapaspadhi254@ gmail.com

Received: 26 January 2017 Accepted in revised form: 27 April 2017 Published online: 24 July 2017 
of cesarean delivery. We screened all apparently healthy neonates (within first 28 days of life) attending the hospital on a fixed day schedule in a week. Thus on the day of screening the pool of babies were from the ones delivered vaginally on the same day or day earlier, and babies delivered by caesarean section in previous 3 days. Parental consent was obtained prior to examination. Babies older than 4 weeks and too sick to undergo the screening examination (purely on the discretion of the pediatrician) were excluded from the study. The Institutional Review Board (2014-1-EM-1) of LV Prasad Eye Institute, Bhubaneswar approved the study and it followed and adhered to the tenets of the declaration of Helsinki. As it was a pilot study, a fixed sample size was not required. This screening protocol was set aside from the regular ROP screening.

The data included gestational age (GA), birth weight (BW), gender, post- menstrual age at examination (PMA), mode of delivery (vaginal, caesarean section, forceps or vacuum assistance if any), significant perinatal events (if any) and contact number. The steps of protocol included the following: (1) parental counseling about the examination procedure and consent, (2) pupillary dilation with a mixture of $2.5 \%$ phenylephrine hydrochloride and $0.5 \%$ cyclopentolate eye drops, (3) entry of relevant data in the WFDRI system data base, (4) application of topical anesthetic eye drops (Proparacaine Hydrochloride 0.5\%), (5) insertion of eye speculums to open the eye and provide adequate exposure for photography in both eyes, (6) assessment of red reflex with the help of the illumination from the Ret Cam 130-degree lens(RetCam II, Clarity medical system, Pleasanton, CA, USA), (7) imaging of the external structures of both eyes including eye lids and anterior segments of each eye, and (8) fundus imaging. Entire fundus was imaged with five basic photographs with the help of RetCam $130^{\circ}$ lens with inert lubricating jelly (K-Y gel) to couple the digital camera lens to the infant's cornea. The five fundus photographs included the posterior pole, including disc and fovea, superior retina-optic disc at the inferior pole of the field of view, inferior retina-optic disc at the superior pole of the field of view, temporal-optic disc at the nasal most part of field of view and nasal retina- optic disc at the temporal most part of the field of view. In addition, the superotemporal, inferotemporal, inferonasal, superonasal quadrant retina were also imaged when required. This was repeated for the fellow eye as well. Topical antibiotic (Tobramycin sulfate $0.3 \%$ ) was instilled in each eye at the conclusion of the procedure. Retinal imaging was deferred in babies with red eye and was confined to red reflex test and external photography only. All imagings were done by an optometrist adequately trained to using RetCam and was aware of the safety precautions while handling these babies. The babies were monitored by a standby nurse. The captured images were analyzed by an ophthalmic resident at the end of the day, the findings were documented in an excel sheet along with other patient details and they were communicated to the parents. When in doubt, the parents were encouraged to bring the baby to the tertiary eye care facility with the concerned specialist ophthalmologist. Images of babies with a positive finding were re-evaluated and crosschecked by a pediatric retina specialist.

The data analysis was done using Statistical Package for Social Sciences (SPSS) version 21.0 (IBM Corp., Armonk, NY, USA). The mean and standard deviations (SD) were calculated for the various parameters (birth weight, gestational age, and so on). Kolmogorov-Smirnov test was used to test the normality of data. Qualitative variables were correlated using chi-square test. A $P$-value of $<0.05$ was considered statistically significant. The expenses involved in terms of machines, transport to the unit, gadgets involved, staff salaries, vis-a-vis the financial benefits in terms of the expenses saved by preventing blindness were evaluated to assess the net monetary gain.

We did a meta analysis of outcome of 3 newborn eye screening studies of similar kind reported so far in the literature and compared it with the present study (Table 2).

\section{Results and discussion}

A total of 1152 babies were examined in the study period and included 598 male (51.9\%) and 554 female babies (48.1\%). The average time to examination was 3.68 days (median: 3.08 days; range 0-21 days). The mean GA and BW were 39.07 weeks (SD: 1.19) and $2.88 \mathrm{~kg}$ (SD: 0.46) respectively. Majority of babies were delivered by LSCS (Lower segment caesarean section; 903; 78.4\%) and the rest by normal vaginal delivery. Ocular abnormality of any kind was seen in 172 babies (14.9\%). Table 1 lists the significant ocular findings. The retinal hemorrhages were the major finding $(153,13.28 \%)$; it was of varying severitysuperficial, subhyaloid and vitreous hemorrhages (Figure 1). The majority (77\%) of hemorrhages were bilateral and about one fifth of them had Roth spots. The hemorrhage was located at fovea in four babies. In majority of cases, the hemorrhages were distributed in all quadrants with varying severity with a tendency to be more around the optic nerve and along the retinal vessels.

Table 1 Significant ocular findings

\begin{tabular}{lc}
\hline Retinal hemorrhages of varying severity & 153 \\
Congenital glaucoma & 02 \\
Cystic fovea & 03 \\
Uveal coloboma & 02 \\
Albinotic fundus & 10 \\
Ridge mimicking Retinopathy of Prematurity & 02 \\
Fundal jaundice & 13 \\
\hline
\end{tabular}




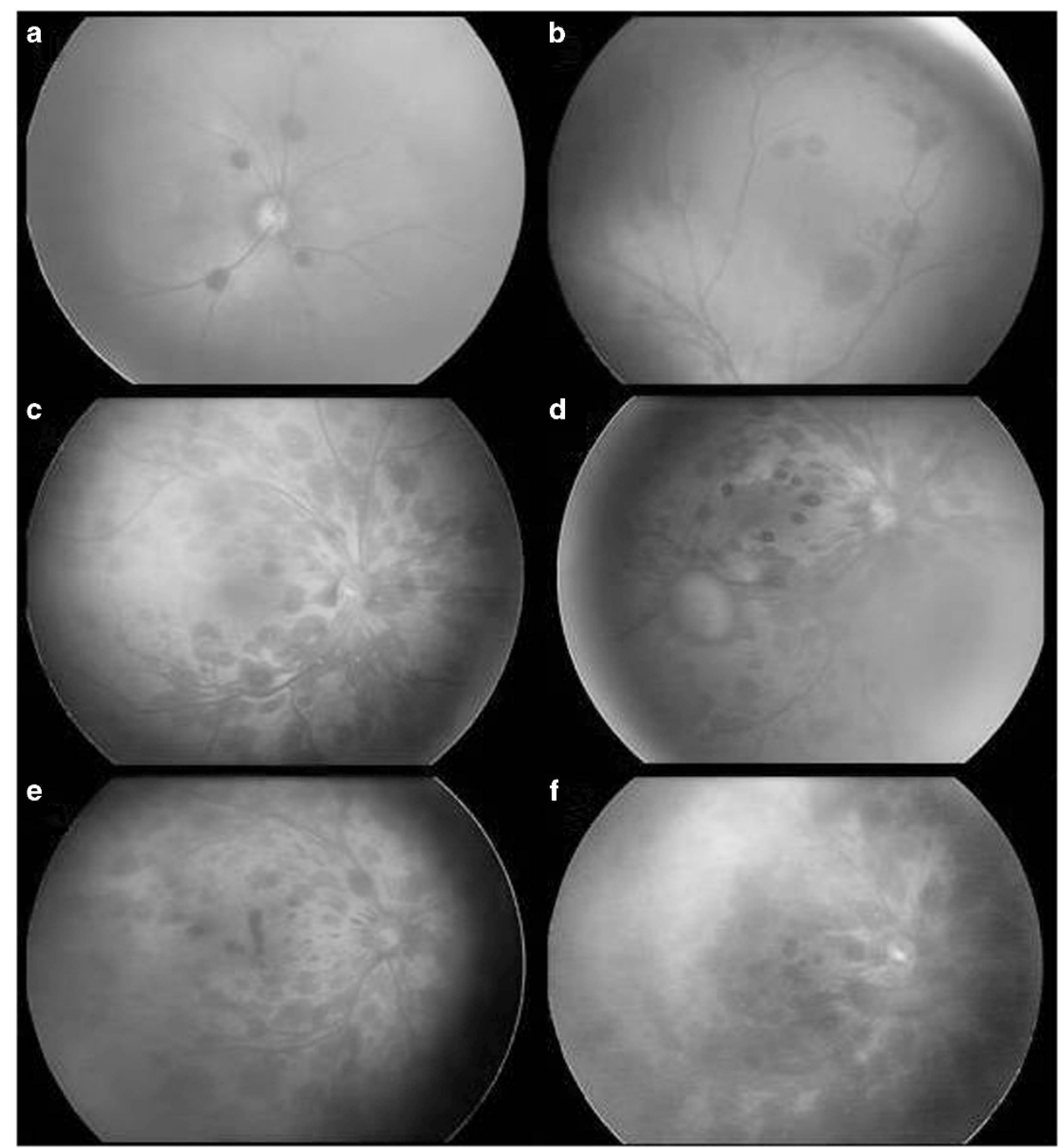

Figure 1 Spectrum of retinal hemorrhages showing few hemorrhage around the disc (a), distribution as far periphery as ora (b), extensive hemorrhage with roth spots (c), pre-retinal hemorrhage ( $d$ and f), additional foveal hemorrhage (e and f).

Retinal hemorrhages were seen in $47.6 \%$ of babies born by normal vaginal delivery and $5.2 \%$ born by LSCS. There was no significant hematological abnormality in those who received blood investigations. While $50 \%$ of them returned for a follow up examination, the rest did not return for various reasons. Those who were compliant to follow up schedules showed complete resolution of hemorrhages within 2 to 6 weeks.

Two babies with enlarged and hazy cornea were diagnosed to having congenital glaucoma (Figure 2, Top left), and treated appropriately. Uveal coloboma

(Figure 2, Top right) was seen in two babies (unilateral in one, bilateral in the other) and the iris was spared in one with unilateral involvement. Both of them were kept under close follow up to evaluate for refractive error, amblyopia and were suggested for barrage laser later in life to reduce the risk of retinal detachment. Two babies showed ridge akin to the one seen in ROP (Figure 2, Bottom left) and were evaluated in detail to rule out other ROP mimickers including FEVR; the ridge regressed with time in one baby and the other baby was lost to follow up. The family members of these babies also received fundus evaluation and did not reveal any significant abnormality. Three babies had cystic fovea (Figure 2, Bottom right); all of them were advised topical carbonic anhydrase inhibitors; the cysts were found resolved (by repeat imaging with RetCam) in all within 1-2 months despite the fact that the medicine was not used in 1baby. We imaged these babies with both 130-degree as well with the high magnification 30-degree lens. We could not access the level of localization of these cysts because of unavailability of Pediatric OCT with us.

Other additional ocular findings included subconjunctival hemorrhage, vascular dilatation, hypo 


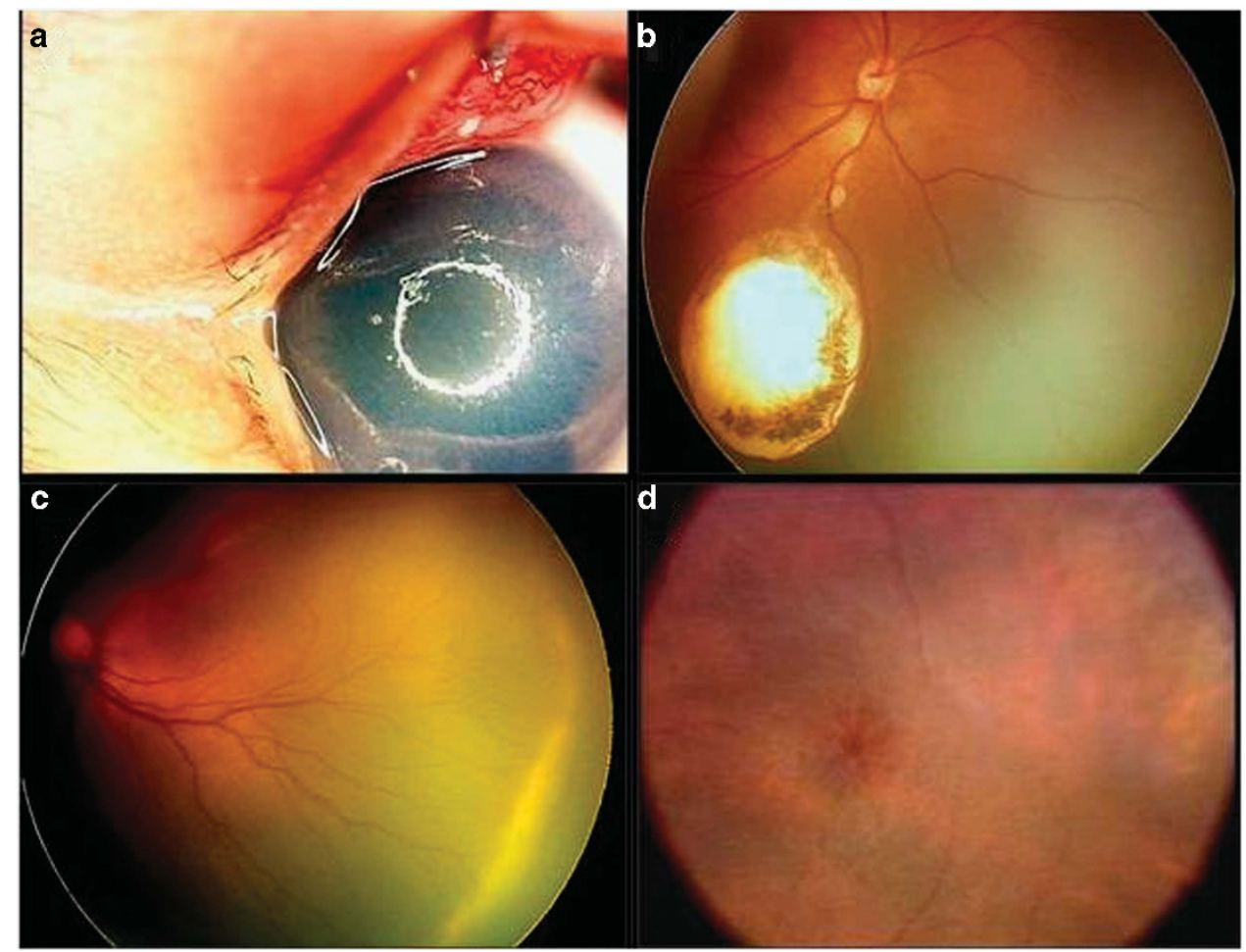

Figure 2 Showing other significant ocular abnormalities. (a) Hazy cornea in a child with congenital glaucoma, (b) Uveal coloboma, (c) Peripheral ridge akin to retinopathy of prematurity, (d) Cystic fovea.

pigmented fundus, immature retina and mature retina with a greenish hue (fundal jaundice). None of the babies had any significant adverse events after the examination. We calculated the total expenses on use of equipment, manpower, stationeries and other accessories, which amounted to INR 845000 (US\$ 12612 @ 1 US\$=INR 67.00; Supplementary Table 1). The RetCam was used for pediatric retinal imaging by various sub specialties for 6 days a week. The cost of RetCam use was 57\% of the entire cost though it was used only for a day for our project. Early disease detection in this project helped 7 babies either directly or indirectly, and at least the baby with glaucoma was saved from irreversible blindness. Considering an average life expectancy of an Indian at 70 years ${ }^{4}$ and the minimum average monthly per capita income at INR 3000 (US\$ 45.00) and the extra living cost of maintaining life of a blind person at another INR $3000 /$ month, we calculated the financial loss incurred due to a child going blind at INR 5.04 million (US\$ 75 224) (Supplementary Table 2). So the net monetary gain in the project was INR4.195 million (US\$ 62 612). We understand that the margin of benefit could have been better with a cheaper pediatric retina camera. We also calculated the amount of time saved by screening on site by a photographer/an ophthalmology resident and reading of the images later by a pediatric retina specialist.
This gain was $319.4 \mathrm{~h}$ or 35.5 working days (Supplementary Table 3).

Quite similar to the observation by other investigators we found a high incidence of retinal hemorrhages in otherwise healthy babies following normal vaginal deliveries. ${ }^{5-10}$ Venous stasis caused by raised intracranial pressure secondary to mechanical head compression while passing through the birth canal is postulated to be the cause for such hemorrhages. ${ }^{7}$ Studies and major reviews on newborn retinal hemorrhages show that they are normal phenomena after birth and can range from 2 to $50 \%$ and majority of them resolve by 6 weeks, so also in the present series (including foveal hemorrhage).

Observation of these hemorrhages therefore should not create over anxiety among parents or create a negative impression on spontaneous vaginal deliveries. We also documented ROP-like retinopathy in term babies similar to one study from India; ${ }^{6}$ the pathogenesis of which is still unexplained. These babies characteristically had avascular retina beyond ridge and showed these findings within a week after birth. This was nearly similar to our previous report on term and near term baby retinopathy. ${ }^{11}$

The cystic fovea seen in these babies has not been reported by any of the 3 other newborn screening studies analyzed in this report though, some other studies have reported similar transient macular cysts documented in 


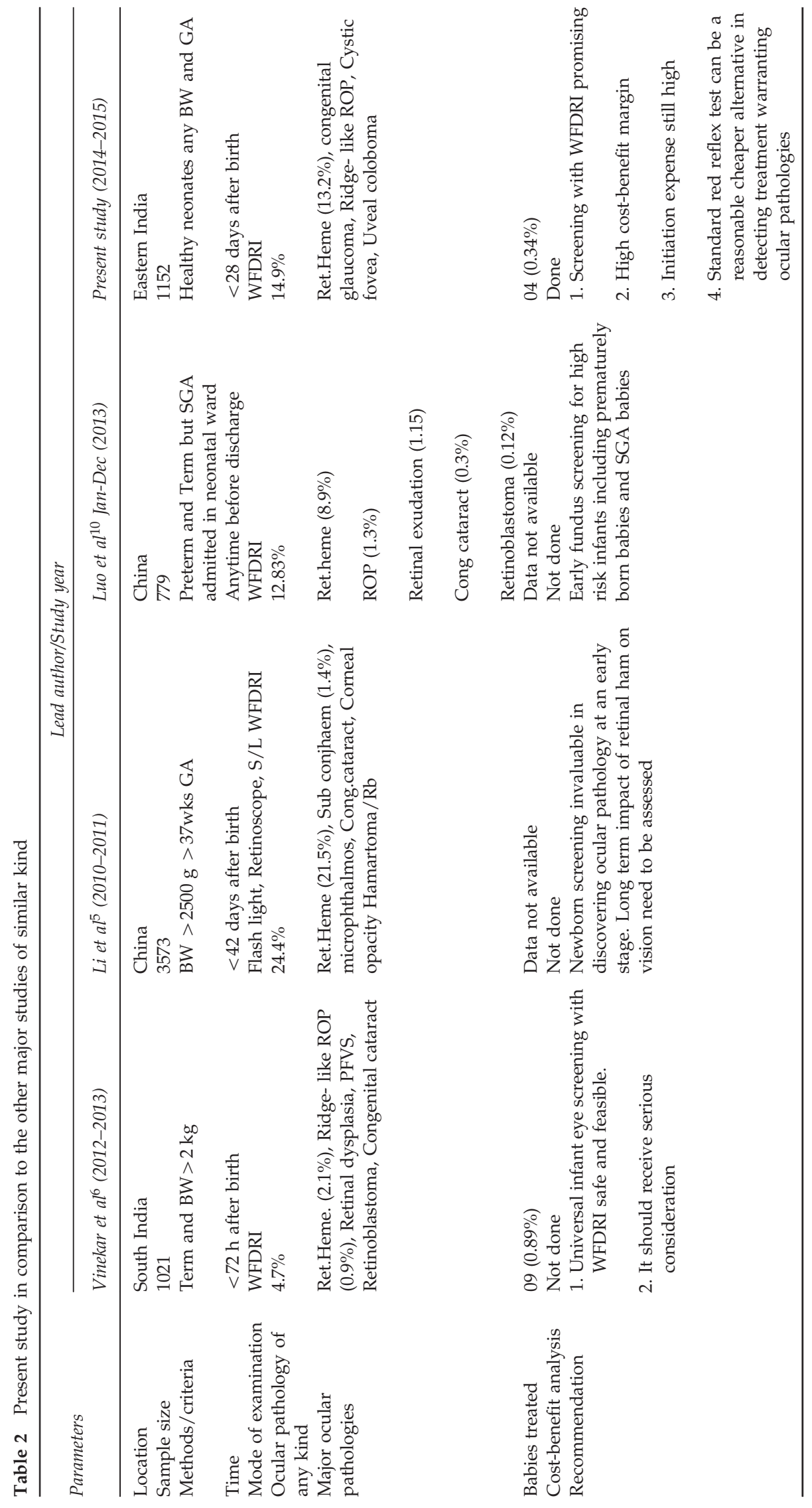


SDOCT in healthy infants. ${ }^{12-14}$ In fact, we have seen similar finding in 6 babies screened for ROP under a separate project (unpublished data). These cysts are hypothesized to be either due to a developmental incompetence of the blood-retinal barrier or may represent non CME transient spaces created during normal migration of retinal cells. ${ }^{14}$ The effect of these cysts on visual development are still unknown and needs further research.

We understand that a red reflex test or just an external eye examination could have detected many of the abnormalities in cumulative 6525 babies documented in the 4 studies (Table 2) cited by us. There were many other retinal pathologies where a late detection could not have mattered. Detection of posterior uveitis mentioned in one group was surprisingly high and it would have been interesting to know the pathologies and if intervention made any difference in their study. ${ }^{6}$ We felt that the 2 cases of early retinoblastomas were the only significant ocular pathologies that could have been missed by external examination/red reflex test alone. One could thus argue that the universal newborn screening studies conducted so far seem to benefit screening for retinoblastoma in the newborns and detection of small choroidal colobomas without iris involvement; other pathologies regress spontaneously, or at best, are of research interest only. Previous reports mention that the most common manifesting sign of children diagnosed to having retinoblastoma in the first month of life is family history. ${ }^{15}$ So looking at the expenses involved one need to re think if universal newborn screening with WFDRI merits over an inexpensive red reflex test of all newborn within the first month of life and restrict retinal examination within a month for high risk groups only (like family history of heritable ocular cancers, FEVR, Premature birth and so on)

As this was a pilot study, we did not set a limit for the sample size. While the cohort size was big enough to detecting common pathologies like retinal hemorrhages, we need a bigger sample size for detection of rare pathologies like intraocular retinoblastomas. The compliance to follow up in normal newborns with retinal hemorrhages in this series was very poor and hence we could not document their pattern of regression completely. Though this study did not aim at, the impact of foveal hemorrhages on visual development could have given us additional information.

Screening is commonly instituted for identifying a previously unrecognized disease or defect in apparently healthy or asymptomatic persons. Often it aims at offering presymptomatic treatment to those so identified. In ideal circumstances where cost of machine and repeat examination is not an issue, the use of WFDRI system in detecting each and every retinal finding could be promising; this saves skilled manpower with a net monetary gain. In the present study, the majority of the findings were retinal hemorrhages which usually do not require treatment and the ones that required treatment could have been detected by a routine red reflex test too. So providing screening for cases that need no intervention with costly camera-like WFDRI system raises question on its affordability.In such an instance, a strict adherence to a routine red reflex test by a neonatologist or pediatrician within 1 month of birth could serve the purpose.

\section{Summary}

What was known before

- There are many newborn eye diseases that present early in life and are actionable at birth but get missed because of the lack of a universal newborn eye screening strategy.

- Photodocumentation by a technician and reading later by a remote expert has already proved itself as an effective telescreening tool in early detection in diabetic retinopathy and retinopathy of Prematurity.

What this study adds

- Universal newborn eye screening with wide-field digital retinal imaging is promising and saves skilled manpower with a net monetary gain in detecting ocular pathology of any kind.

- However, when cost is a concern and we aim at detecting pathologies that could have been missed by a simple red reflex test, the benefits were found to be marginal compared to the expenses involved. In such an instance, a strict adherence to a routine red reflex test by a neonatologist or pediatrician within 1 month of birth could serve the purpose.

\section{Conflict of interest}

The authors declare no conflict of interest.

\section{Acknowledgements}

The LV Prasad eye institute receives funding for ROP related programs from Orbis international, Sight savers International, Queen Elizabeth Diamond Jubilee Trust, Miriam Hyman Children's Eye Care Center and other philanthropists. These funding agencies are not involved directly in the present study and none of the authors get paid directly by them.

\section{References}

1 Castillo-Riquelme MC, Lord J, Moseley MJ, Fielder AR, Haines L. Cost-effectiveness of digital photographic screening for retinopathy of prematurity in the United Kingdom. Int J Technol Assess Health Care 2004; 20(2): 201-213. 
2 Martínez Rubio M, Moya Moya M, Bellot Bernabé A, Belmonte Martínez J. Diabetic retinopathy screening and teleophthalmology. Arch Soc Esp Oftalmol 2012; 87(12): 392-395.

3 Jones S, Edwards RT. Diabetic retinopathy screening: A systematic review of the economic evidence. Diabet Med 2010; 27(3): 249-256.

4 Census India. Govt of India Ministry of Home AffairsCensus report. Compendium of India's Fertility and Mortality Indicators, based on SRS,1971-2013. Accessed at http://www.censusindia.gov.in/vital_statistics/ Compendium/Srs_data.html. on 9 Dec 2016.

5 Li L-H, Li N, Zhao JY, Fei P, Zhang GM, Mao JB et al. Findings of perinatal ocular examination performed on 3573, healthy full-term newborns. Br J Ophthalmol 2013; 97(5): 588-591.

6 Vinekar A, Govindaraj I, Jayadev C, Kumar AK, Sharma P, Mangalesh S et al. Universal ocular screening of 1021 term infants using wide-field digital imaging in a single public hospital in India - A pilot study. Acta Ophthalmol 2015; 93(5): e372-e376.

7 Callaway NF, Ludwig CA, Blumenkranz MS, Jones JM, Fredrick DR, Moshfeghi DM. Retinal and optic nerve hemorrhages in the newborn infant: One-Year Results of the Newborn Eye Screen Test Study. Ophthalmology 2016; 123: 1043-1052.

8 Kaur B, Taylor D. Fundus hemorrhages in infancy. Surv Ophthalmol 1992; 37(1): 1-17.
9 Zhao Q, Zhang Y, Yang Y, Li Z, Lin Y, Liu R et al. Birthrelated retinal hemorrhages in healthy full-term newborns and their relationship to maternal, obstetric, and neonatal risk factors. Graefes Arch Clin Exp Ophthalmol 2015; 253(7): 1021-1025.

10 Luo R, Liu J, Hu P, Cheng SS, Shi BZ, Zhu JH et al. Results of 779 cases of neonatal fundus screening and risk factors for neonatal fundus diseases. Zhongguo Dang Dai Er Ke Za Zhi 2014; 16(12): 1197-1201.

11 Padhi T, Rath S, Jalali S, Pradhan L, Kesarwani S, Nayak M et al. Larger and near-term baby retinopathy: a rare case series. Eye 2014; 29(2): 286-289.

12 Cabrera MT, Maldonado RS, Toth CA, O'Connell RV, Chen BB, Chiu SJ et al. Subfoveal fluid in healthy full-term newborns observed by handheld spectral-domain optical coherence tomography. Am J Ophthalmol 2012; 153(1): 167-175.e3.

13 Lee AC, Maldonado RS, Sarin N, O'Connell RV, Wallace DK, Freedman SF et al. Macular features from spectral-domain optical coherence tomography as an adjunct to indirect ophthalmoscopy in retinopathy of prematurity. Retina 2011; 31(8): 1470-1482.

14 Ganesh A, Khalighi M, Hammersmith K, Levin AV. Spontaneously resolving macular cyst in an infant. Oman J Ophthalmol 2013; 6(3): 203-205.

15 Abramson DH, Du TT, Beaverson KL, Sciences C. (Neonatal) retinoblastoma in the first month of life. Arch Ophthalmol 2002; 120(6): 738-742.

Supplementary Information accompanies this paper on Eye website (http:/ /www.nature.com/eye) 